\title{
Three-dimensional assessment of the effects of high-density embolization material on the absorbed dose in the target for Gamma Knife radiosurgery of arteriovenous malformations
}

\author{
Yoichi Watanabe, PhD, ${ }^{1}$ Divyajot Sandhu, MD, ${ }^{2-4}$ Leighton Warmington, PhD, ${ }^{1}$ Sean Moen, BA, ${ }^{3,4}$ \\ and Ramachandra Tummala, MD ${ }^{4}$
}

Departments of ${ }^{1}$ Radiation Oncology, ${ }^{2}$ Neurology, ${ }^{3}$ Radiology, and ${ }^{4}$ Neurosurgery, University of Minnesota, Minneapolis, Minnesota

\begin{abstract}
OBJECTIVE Arteriovenous malformation (AVM) is an intracranial vascular disorder. Gamma Knife radiosurgery (GKRS) is used in conjunction with intraarterial embolization to eradicate the nidus of AVMs. Clinical results indicate that patients with prior embolization tend to gain less benefit from GKRS. The authors hypothesized that this was partly caused by dosimetric deficiency. The actual dose delivered to the target may be smaller than the intended dose because of increased photon attenuation by high-density embolic materials. The authors performed a phantom-based study to quantitatively evaluate the 3D dosimetric effect of embolic material on GKRS.
\end{abstract}

METHODS A 16-cm-diameter and 12-cm-long cylindrical phantom with a 16-cm-diameter hemispherical dome was printed by a 3D printer. The phantom was filled with radiologically tissue-equivalent polymer gel. To simulate AVM treatment with embolization, phantoms contained Onyx 18. The material was injected into an AVM model, which was suspended in the polymer gel. The phantom was attached to a Leksell frame by standard GK fixation method, using aluminum screws, for imaging. The phantom was scanned by a Phillips CT scanner with the standard axial-scanning protocol (120 kV and 1.5-mm slice thickness). CT-based treatment planning was performed with the GammaPlan treatment planning system (version 10.1.1). The plan was created to cover a fictitious AVM target volume near the embolization areas with eleven 8-mm shots and a prescription dose of $20 \mathrm{~Gy}$ to $50 \%$ isodose level. Dose distributions were computed using both tissue maximum ratio (TMR) 10 and convolution dose-calculation algorithms. These two 3D dose distributions were compared using an in-house program. Additionally, the same analysis method was applied to evaluate the dosimetric effects for 2 patients previously treated by GKRS.

RESULTS The phantom-based analyses showed that the mean dose difference between TMR 10 and convolution doses of the AVM target was no larger than $6 \%$. The difference for GKRS cases was $5 \%$. There were small areas where a large dose difference was observed on the isodose line plots, and those differences were mostly at or in the vicinity of the embolization materials.

CONCLUSIONS The results of both the phantom and patient studies showed a dose reduction no larger than $5 \%$ due to the embolization material placed near the target. Although the comparison of 3D dose distributions indicated small local effects of the embolic material, the clinical impact on the obliteration rate is expected to be small.

http://thejns.org/doi/abs/10.3171/2016.7.GKS161545

KEY WORDS stereotactic radiosurgery; AVM; arteriovenous malformation; embolization; GammaPlan; TMR 10; convolution; dosimetry

$\mathrm{A}$ RTERIOVENOUS malformation (AVM) is an intracranial vascular disorder that poses risk for intracranial hemorrhage and neurological morbidity. Gamma Knife radiosurgery (GKRS) is used in conjunction with intraarterial embolization to eradicate the nidus of certain AVMs. However, clinical results indicate that patients with prior embolization tend to benefit less from GKRS. ${ }^{1}$ The lower obliteration rate in patients with prior embolization has been attributed to the following 3 factors: recanalization of the embolized AVM, difficult tar-

ABBREVIATIONS AVM = arteriovenous malformation; GKRS = Gamma Knife radiosurgery; NBCA = N-butyl cyanoacrylate; TMR = tissue maximum ratio

SUBMITTED June 10, 2016. ACCEPTED July 21, 2016.

INCLUDE WHEN CITING DOI: 10.3171/2016.7.GKS161545. 
TABLE 1. Dosimetric properties of embolization materials

\begin{tabular}{lccc}
\hline Material & Zeff $^{*}$ & Electron Density, $\rho_{\mathrm{e}}$ (relative to water) & $\mu(1 / \mathrm{cm}) \dagger$ \\
\hline NBCA & & 2.34 & 0.0516 \\
\hline NBCA-Ta & & 2.41 & 0.0580 \\
\hline Onyx 18 & 3.36 & 2.71 & 0.11 \\
\hline Onyx 34 & 2.89 & 0.11 \\
\hline Onyx & & & 0.0784 \\
\hline Water & 3.33 & 1.0 & 0.05 \\
\hline
\end{tabular}

$\mathrm{Ta}=$ tantalum

* For $1.25-\mathrm{MeV}$ photons.

$\dagger$ For 6-MV photon beam.

geting of the embolized AVM for GKRS, and the dose reduction due to the high-density material used for the embolization. ${ }^{1}$

The physical characteristics of the most common liquid embolic agents, $N$-butyl cyanoacrylate (NBCA) and Onyx (ethylene vinyl alcohol copolymer), are shown in Table 1 . The electron densities of those materials are approximately 2.5 times larger than water. The photon attenuation coefficient of Onyx 18 is twice as large as that of water for the 6-MV photon beam. For example, these data imply that a 5-mm-thick layer of Onyx-18 can decrease the dose by $5 \%$. There are several studies in which the dosimetric effects of the embolization materials have been investigated..$^{2,6-8}$ However, there is no dosimetric study showing the 3D effects of the high-density embolic material to the dose distribution for GKRS of AVMs.

In this study, we investigated the effects of the embolization material on the dose to the AVM target with GKRS. We analyzed a phantom including Onyx 18, which simulated the shape and location of a clinically realistic embolization cast, and data of patients who had GKRS and pre-GKRS embolization. The dosimetric impact was assessed by comparing doses calculated by the Leksell GammaPlan treatment planning system for the homogeneous and heterogeneous media. The analytical prediction was correlated to the obliteration rate obtained from clinical data presented by Flickinger et al. ${ }^{4}$ The present study was conducted under institutional review board approval.

\section{Methods \\ Phantom Experiment}

Onyx 18 was injected into an AVM model so that the material distribution mimicked the shape of the realistic embolization cast, as shown in Fig. 1A. A 16-cm-diameter and $12-\mathrm{cm}$-long cylindrical phantom with a $16-\mathrm{cm}$-diameter hemispherical dome was manufactured by a 3D printer. The phantom was filled with a radiologically tissueequivalent polymer gel (normoxic methacrylic acid gel). ${ }^{3}$ The AVM model was suspended in the polymer gel. The phantom was attached to a Leksell frame by the standard Gamma Knife fixation method (using aluminum screws) for imaging. Figure 1B shows the phantom with the Leksell frame.

The phantom was scanned by the Brilliance large-bore CT simulator (Philips Healthcare) using a standard axialscanning protocol (120 kV and $1.5-\mathrm{mm}$ slice thickness). In addition to this CT scan ( $\mathrm{kVCT})$, we also scanned the phantom with the MV CT scanner (MVCT). For this task, we used the imaging mode of TomoTherapy (Accuray). The MVCT scan has the potential to reduce the image artifacts caused by the high atomic number (Z) atoms included in the embolization material.

The CT data were imported into the GammaPlan treatment planning system (version 10.1.1). We drew an AVM target near the embolization material as well as a second structure (Structure B) at the embolization. The treatment plan used eleven 8-mm shots with the prescription dose of 20 Gy to $50 \%$ of the maximum dose inside the target. We

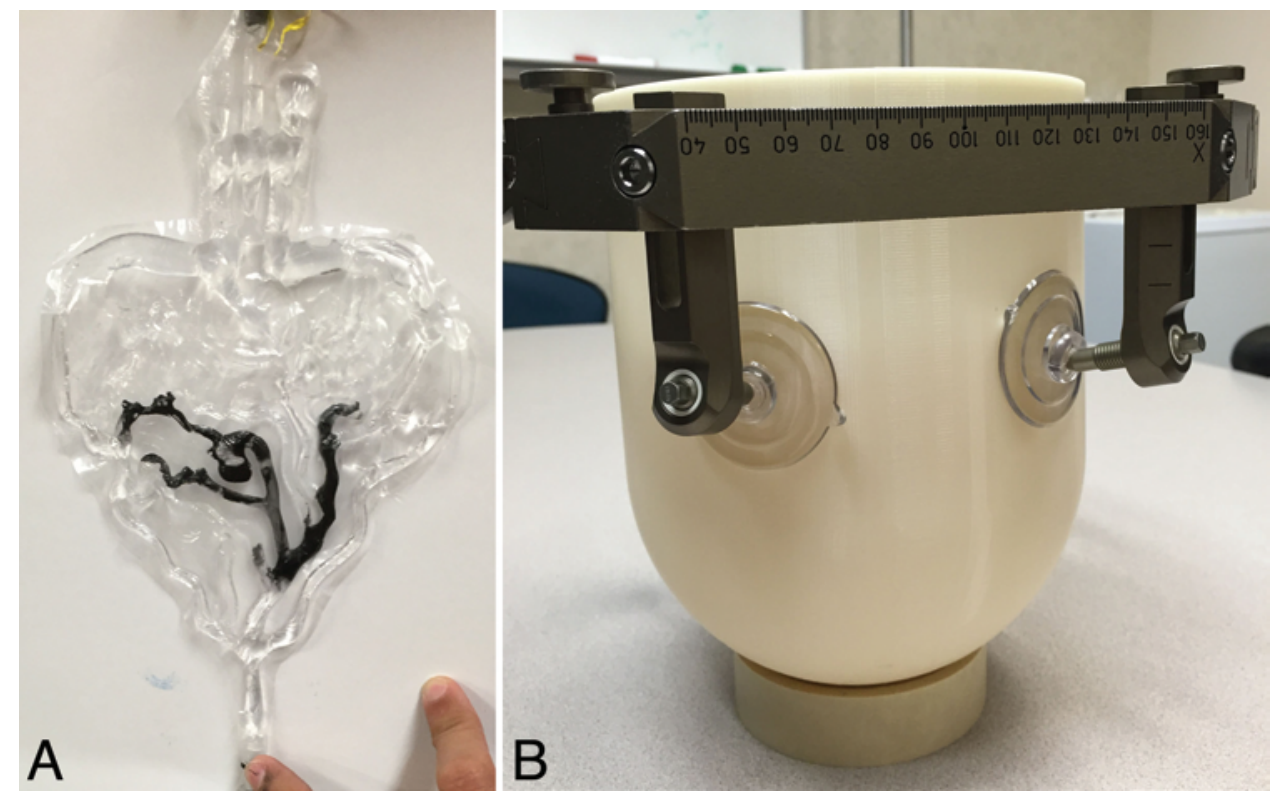

FIG. 1. A: An AVM sample with Onyx 18. B: A 3D-printed phantom. 
TABLE 2. GKRS patient data

\begin{tabular}{cccccc}
\hline Patient & $\begin{array}{c}\text { Target } \\
\text { Vol, } \\
\mathrm{cm}^{3}\end{array}$ & $\begin{array}{c}\text { Dose, } \\
\text { Gy/\% } \\
\mathrm{Rx}\end{array}$ & $\begin{array}{c}\text { Shot, No. } \\
\times \text { Size in } \\
\mathrm{mm}\end{array}$ & $\begin{array}{c}\text { Embolization } \\
\text { Material }\end{array}$ & Outcome \\
\hline A & 0.59 & $20: 50$ & $4 \times 8$ & $\begin{array}{c}\text { NBCA, plati- } \\
\text { num coils }\end{array}$ & Obliterated \\
\hline B & 13.8 & $16: 50$ & $12 \times 14$ & Onyx 18 & $\begin{array}{r}\text { Deceased in } \\
6 \text { months }\end{array}$ \\
\hline
\end{tabular}

$\mathrm{Rx}=$ prescription

calculated the dose using the convolution algorithm along with the CT number-to-density tables of both the kVCT and MVCT scanners.

The dose and image data in the DICOM format were exported to another workstation by turning on either the convolution or tissue maximum ratio (TMR) 10 dosecalculation algorithms for further analysis. We used an in-house PG3DCMP program for 3D comparison of the dose distributions obtained by the TMR 10 (TMR10) and convolution algorithms. The TMR10 dose calculation assumes a homogeneous medium by ignoring the embolization material. On the other hand, the convolution algorithm (Convolution) takes into account the heterogeneity introduced by the embolization material. Hence, the comparison of the dose distributions obtained by TMR 10 and Convolution is equivalent to comparing the effect of the embolization material on the absorbed dose in the medium.

\section{Patient Data}

To evaluate the effects of embolization on the actual patients, we selected 2 patients from our database, Patient $\mathrm{A}$ and Patient B, who had AVM embolization prior to GKRS and had a CT scan after the embolization procedure. The patient data are summarized in Table 2. Because of the small size $\left(0.59 \mathrm{~cm}^{3}\right)$ of the AVM of Patient A, the prescription dose was 20 Gy to $50 \%$ of the maximum dose inside the target with four 8-mm shots. Prior to the GKRS, this patient had staged embolization in 2 regions of the AVM nidus, one in the anterior right side of the target with NBCA and another at the posterior left side with platinum coils. The patient had a follow-up CT scan 4 months after the GKRS. For the current analysis, the follow-up CT data were coregistered with the treatment MR image. The dose calculation was done with the CT data.

Patient B had a large AVM of $13.8 \mathrm{~cm}^{3}$. The plan used twelve $14-\mathrm{mm}$ shots with 16 Gy to $50 \%$ of the maximum dose inside the target. This patient had an embolization using Onyx 18 prior to the GKRS. The AVM target of GKRS was located posterior to the Onyx 18 regions. Note that Patient A had a complete obliteration, but Patient B died 6 months after GKRS due to complications resulting from urosepsis.

\section{Results}

\section{Phantom Study}

Table 3 shows the comparison of volumetric doses between TMR10 and Convolution by using $\mathrm{kVCT}$ and
TABLE 3. Dosimetric effects of Onyx 18 in the phantom: kVCT versus MVCT

\begin{tabular}{|c|c|c|c|c|c|c|}
\hline \multirow[b]{2}{*}{ Target } & \multicolumn{3}{|c|}{ kVCT Dose } & \multicolumn{3}{|c|}{ MVCT Dose } \\
\hline & Min & Max & Mean & Min & Max & Mean \\
\hline \multicolumn{7}{|l|}{ AVM } \\
\hline TMR10, Gy & 15.4 & 41.0 & 30.2 & 15.2 & 40.8 & 30.0 \\
\hline Convolution, Gy & 15.0 & 40.4 & 29.5 & 14.3 & 39.1 & 28.3 \\
\hline$\%$ Difference & -2.6 & -1.5 & -2.3 & -5.7 & -4.2 & -5.8 \\
\hline \multicolumn{7}{|l|}{ Structure B } \\
\hline TMR10, Gy & 7.9 & 29.9 & 13.3 & 7.9 & 29.7 & 13.3 \\
\hline Convolution, Gy & 0.0 & 29.2 & 12.6 & 7.3 & 28.1 & 12.3 \\
\hline$\%$ Difference & & -2.3 & -5.2 & -7.3 & -5.5 & -7.2 \\
\hline
\end{tabular}

MVCT data. The mean AVM doses of Convolution were $2.3 \%$ and $5.8 \%$ lower than TMR10 for KVCT and MVCT, respectively. The mean doses of the Structure B of Convolution were lower than TMR10 by $5.2 \%$ and $7.2 \%$ for $\mathrm{kVCT}$ and MVCT, respectively. The differences of the maximum AVM and Structure B doses between TMR10 and Convolution showed the same trend as the mean doses. The minimum dose of the Structure B by Convolution with $\mathrm{kVCT}$ was 0 and it differed greatly from the TMR10 value. Such a large difference was not seen with the MVCT data.

The effects of the Onyx 18 material on the local dose were seen when the dose distributions of TMR10 and Convolution were displayed on the same plots of different transverse planes. Figure 2A shows the isodose plots for kVCT and Fig. 2B shows those for MVCT. Overall, these showed a very small difference between TMR 10 and Convolution, but there are a few areas where there are noticeable distortions of isodose lines due to the presence of Onyx 18.

\section{Patient Data}

The dosimetric data of Patients A and B are presented in Table 4. The mean dose differences between TMR10 and Convolution were $6.0 \%$ and $5.5 \%$ for Patients $\mathrm{A}$ and $\mathrm{B}$, respectively. The maximum dose shows that the Convolution dose is smaller by $4.7 \%$ than the TMR 10 value. The minimum dose of Patient A was 0 by Convolution because the AVM target encompassed the embolized area.

There are a few areas where the isodose distributions were significantly different between TMR10 and Convolution. These plots are presented in Fig. 3A for Patient A and in Fig. 3B for Patient B. These differences at low dose levels were caused by the presence of the embolization material in those areas.

\section{Discussion}

This study shows that the effects of embolization materials on the mean dose of the AVM target are approximately a 5\% reduction for both the phantom AVM model and 2 actual patients. The local dosimetric effects of the embolization material are larger than 5\%, but those are in small areas and limited to areas close to the high-density embolization material. 

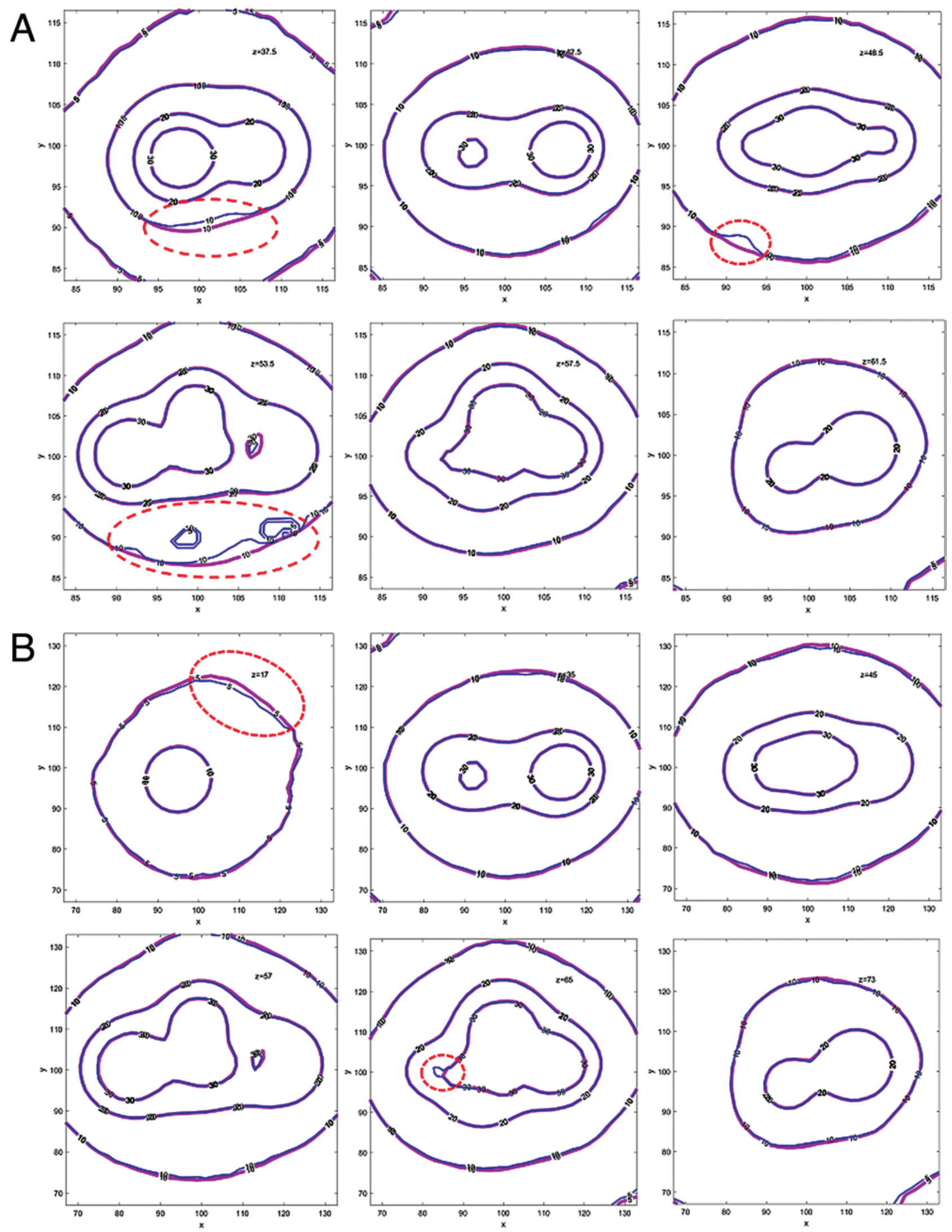

FIG. 2. Isodose line plots of the phantom plan; red lines for TMR10 and blue lines for Convolution. A: kVCT. B: MVCT. 
TABLE 4. Dosimetric comparison of GKRS patient cases

\begin{tabular}{|c|c|c|c|}
\hline Patient & Min Dose & Max Dose & Mean Dose \\
\hline \multicolumn{4}{|l|}{$A$} \\
\hline TMR10, Gy & 13.7 & 34.1 & 23.5 \\
\hline Convolution, Gy & 0.0 & 32.5 & 22.1 \\
\hline$\%$ Difference & -13.7 & -4.7 & -6.0 \\
\hline \multicolumn{4}{|l|}{ B } \\
\hline TMR10, Gy & 17.1 & 42.7 & 29.3 \\
\hline Convolution, Gy & 16.2 & 40.7 & 27.7 \\
\hline$\%$ Difference & -5.3 & -4.7 & -5.5 \\
\hline
\end{tabular}

Two previous studies have investigated the dosimetric effects of the embolization materials. Mamalui-Hunter et al. performed measurements in a phantom containing Onyx, irradiated with GKRS, and found that the dose reduction due to the material was negligible. Their results were supported by a theoretical estimation. ${ }^{7}$ Bing et al. constructed AVM models including Onyx 18 and placed them in plastic phantoms. They generated a 4-arc stereotactic treatment plan with a 6-MV photon beam. Their measurement results showed no dosimetric reduction due to the embolization material. ${ }^{2}$ Our results are in accordance with their results if the systematic dose difference between TMR10 and the convolution algorithms is considered.

There are some factors that limit the accuracy of the current approach for the evaluation of the dosimetric effects of the embolization material. First, we compared the doses calculated by the TMR10 and convolution algorithms by assuming that the latter algorithm can accurately model the effects of the heterogeneity created by the high-density material. Second, we thought that the image artifacts of the CT image data caused by the high-density material on the dose calculations are negligible. This assumption was partially justified when we examined the dosimetric difference between the dose calculations done with KVCT and MVCT data. We saw an apparent reduction of the image artifacts on the MVCT data, and the dosimetric comparisons showed that the embolization material (Onyx 18) resulted in 5.8\% reduction of the mean target dose. This was similar to the difference of $2.3 \%$ with the kVCT data.

To address the first assumption on the accuracy of the convolution algorithm, further study is needed by using more accurate dose-calculation methods (such as the Monte Carlo simulation) and doing actual dose measurements with 3D dosimeters (such as polymer gel dosimeters). Such studies may delineate the accuracy of the dose calculations. There are several studies showing the systematic dose difference between the doses predicted by TMR 10 and Convolution. ${ }^{5,9}$ Even though there is no clear explanation for the difference, one potential reason is attributed to the difference in the medium. The TMR10 assumes pure water, whereas the Convolution uses the material density, which is estimated from the CT image with the CT number-to-density conversion data. The dose difference observed in our study may be explained by this. If so, the global effect of the embolization is even smaller.

Although not specific to the AVM GKRS, there is 1 factor that may cause the dose reduction to the target when a frame-based technique is used. For the GKRS, we use 4 sets of posts and screws to secure the Leksell GK frame to the head. The high-density metal (approximately 3.2$3.7 \mathrm{~g} / \mathrm{cm}^{3}$ ) of the holding pins may attenuate the photons passing through those parts by up to $10 \%$. To minimize the effects of those metallic parts, we placed the target so that the number of beams interfering with the posts and screws are minimal. With the patient treatment, we found that anterior positioning of the posts and screws relative to the AVM target is superior to those positioned posteriorly. Therefore, the beam-attenuation effects are negligible for the sample cases analyzed in our study. However, it is noted that the effects of those metallic materials on the target dose should be considered if a large number of beams pass through those parts.

There are an infinite number of possible spatial arrangements of the AVM target and the embolization material. For the current study, we investigated 1 arrangement
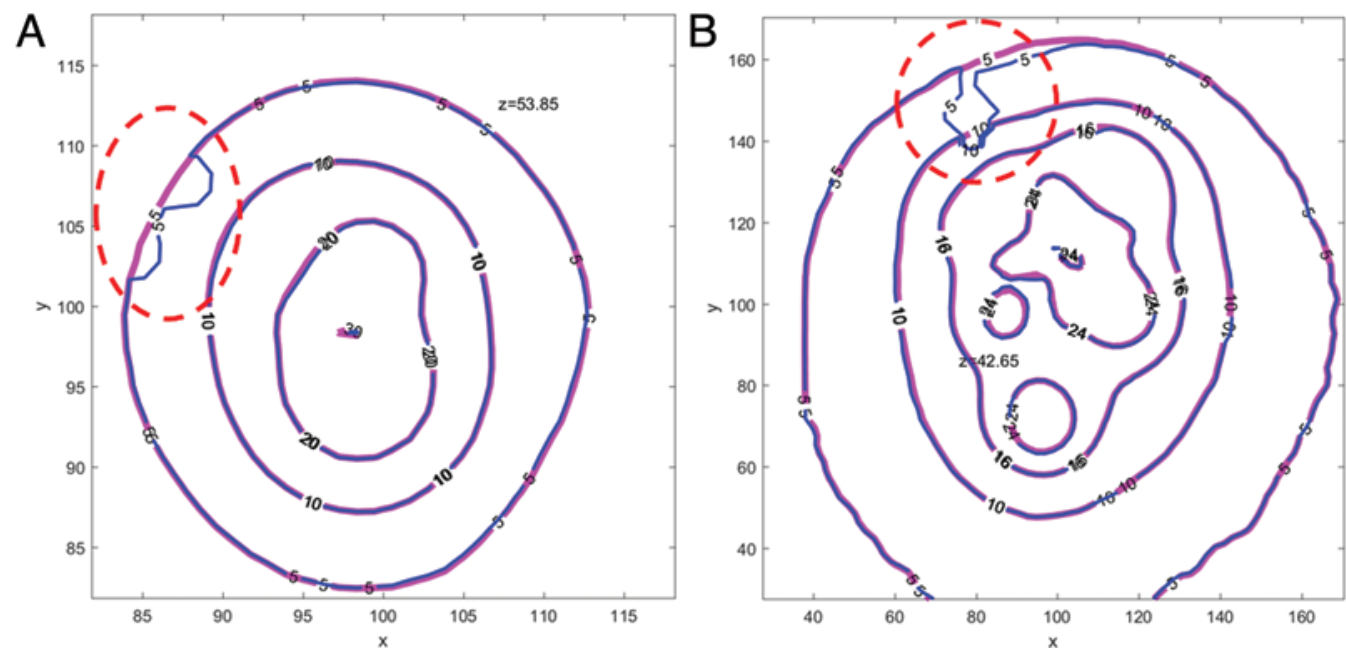

FIG. 3. Isodose line plots of the patient plans; red lines for TMR10 and blue lines for Convolution. A: Patient A. B: Patient B. 


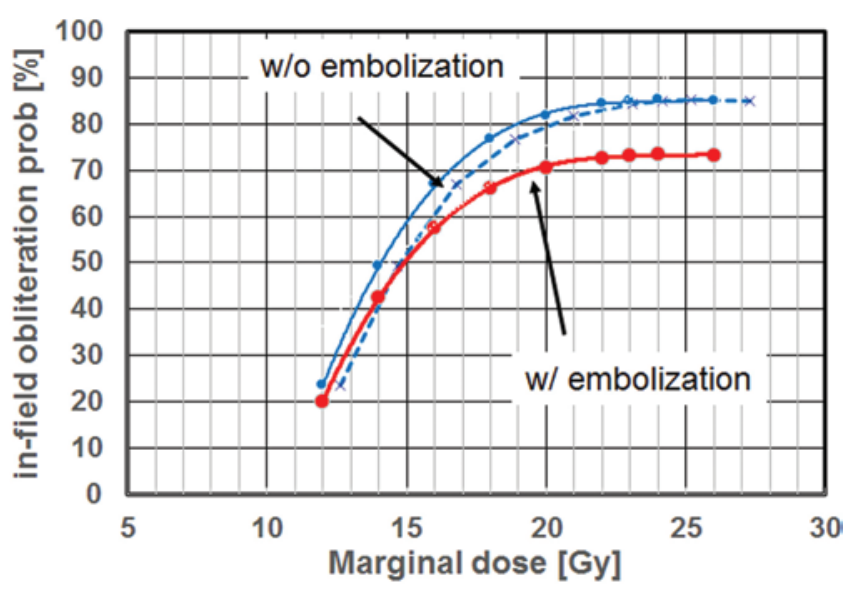

FIG. 4. Radiobiological effects of embolization on the obliteration probability as a function of the dose. The figure was plotted based on the data published in the article by Flickinger et al. ${ }^{4}$ prob $=$ probability.

for a phantom and 2 actual patients. There are strong indications that the dose distribution could be largely altered by high-density material even though it would cover only a small part of the total target volume. Therefore, we recommend the Convolution dose calculation using CT image data for patients with prior embolization for more accurate assessment of the dosimetric effects of the embolic material.

With the small dosimetric effects observed in our study, it is important to evaluate if the observed difference can explain the effect on the obliteration rate clinically seen with patients. Figure 4 presents the obliteration probability as a function of the marginal dose to the AVM target. ${ }^{4}$ The blue solid line is the response curve for the patients without prior embolization, and the red solid line is the curve for the patients with prior embolization. These curves indicate that the embolization reduces the probability of obliteration even if the same dose was used for the treatment. When the actual dose delivered to the target is smaller than the planned dose by $5 \%$ due to the embolization material, the obliteration probability decreases, as indicated by the dotted curve in Fig 4. The curve shows that the probability decreases by $6 \%$ for 16 Gy and $2 \%$ for 20 Gy. It is noteworthy that the GKRS treatment of Patient $\mathrm{B}$, who received only a $16-$ Gy marginal dose, might have shown failure if longer follow-up was possible.

\section{Conclusions}

The dosimetric effects of the embolization (NBCA, platinum coils, Onyx 18) to the target mean dose is $<6 \%$ for GKRS. The comparison of 3D dose distributions indicated only small local effects of the embolic material, which resulted in cold areas. Therefore, we believe that the main cause of lower AVM obliteration rates of patients who underwent embolization is not the dosimetric effect.
In the future, we plan to confirm the theoretical prediction by using polymer gel dosimeter.

\section{References}

1. Andrade-Souza YM, Ramani M, Scora D, Tsao MN, terBrugge K, Schwartz ML: Embolization before radiosurgery reduces the obliteration rate of arteriovenous malformations. Neurosurgery 60:443-452, 2007

2. Bing F, Doucet R, Lacroix F, Bahary JP, Darsaut T, Roy D, et al: Liquid embolization material reduces the delivered radiation dose: clinical myth or reality? AJNR Am J Neuroradiol 33:320-322, 2012

3. De Deene Y, Vergote K, Claeys C, De Wagter C: The fundamental radiation properties of normoxic polymer gel dosimeters: a comparison between a methacrylic acid based gel and acrylamide based gels. Phys Med Biol 51:653-673, 2006

4. Flickinger JC, Kondziolka D, Maitz AH, Lunsford LD: An analysis of the dose-response for arteriovenous malformation radiosurgery and other factors affecting obliteration.

Radiother Oncol 63:347-354, 2002

5. Hundertmark B, Watanabe Y: Validation of the Leksell GammaPlan convolution-based dose calculation algorithm. Med Phys 40:332, 2013

6. Labby ZE, Chaudhary N, Gemmete JJ, Pandey AS, Roberts DA: Dosimetric measurements of an n-butyl cyanoacrylate embolization material for arteriovenous malformations. Med Phys 42:1739-1744, 2015

7. Mamalui-Hunter M, Jiang T, Rich KM, Derdeyn CP, Drzymala RE: Effect of liquid embolic agents on Gamma Knife surgery dosimetry for arteriovenous malformations. Clinical article. J Neurosurg 115:364-370, 2011

8. Roberts DA, Balter JM, Chaudhary N, Gemmete JJ, Pandey AS: Dosimetric measurements of Onyx embolization material for stereotactic radiosurgery. Med Phys 39:66726681, 2012

9. Xu A, Bhatnagar J, Bednarz G, Niranjan A, Flickinger J, Lunsford LD, et al: Dose differences between the three dose calculation algorithms in Leksell GammaPlan. J Appl Clin Med Phys 15: 89-99, 2014

\section{Disclosures}

The authors report no conflict of interest concerning the materials or methods used in this study or the findings specified in this paper.

\section{Author Contributions}

Conception and design: Watanabe, Sandhu, Moen, Tummala. Acquisition of data: Watanabe, Sandhu, Warmington, Moen. Analysis and interpretation of data: Watanabe, Sandhu. Drafting the article: Watanabe, Sandhu. Critically revising the article: Watanabe. Reviewed submitted version of manuscript: Watanabe. Approved the final version of the manuscript on behalf of all authors: Watanabe. Administrative/technical/material support: Watanabe, Sandhu, Moen, Tummala. Study supervision: Tummala.

\section{Correspondence}

Yoichi Watanabe, Department of Radiation Oncology, University of Minnesota, 420 Delaware St. SE, MMC-494, Minneapolis, MN 55455.email: watan016@umn.edu. 